\title{
Profound Amnesia after Temporal Lobectomy: An Autoimmune Process Resembling Patient H.M.?
}

\author{
Michael Bonello Andrew J. Larner Anthony G. Marson \\ Walton Centre for Neurology and Neurosurgery, Liverpool, UK
}

\section{Key Words}

Amnesia - Epilepsy - Glutamic acid decarboxylase - Limbic encephalitis · Temporal lobectomy

\begin{abstract}
We describe a patient who developed significant cognitive decline with profound amnesia following non-dominant temporal lobectomy for refractory seizures, in whom the original suspicion of structural pathology was revised following the discovery of clinical and neuropathological markers of inflammation, neuropsychological evidence of bilateral involvement, and high titres of antibodies directed against glutamic acid decarboxylase (GAD). This case adds to the evidence that the diagnosis of non-paraneoplastic anti-GAD limbic encephalitis merits consideration in any patient with a refractory seizure disorder and cognitive decline.

(c) 2014 S. Karger AG, Basel
\end{abstract}

\section{Introduction}

Surgical resections such as unilateral temporal lobectomy and amygdalo-hippocampectomy have an established place in the management of carefully selected patients with refractory localisation-related epilepsy. Adverse cognitive sequelae of epilepsy surgery have been well-recognised since the seminal report of Scoville and Milner in 1957 documenting the dense anterograde amnesia in patient H.M. following bilateral anterior temporal lobectomy involving hippocampal structures [1]. Such amnesia has been observed on occasion following unilateral surgery, reflecting preoperative damage in the unoperated, contralateral, temporal lobe [2], a finding which mandates careful preoperative assessment of the non-operated hemisphere, for example using sodium amytal (Wada) testing and/or functional neuroimaging, to try to ensure cognitive function is preserved post-operatively. 
We present a patient with refractory epilepsy who, following initially apparently successful unilateral temporal lobectomy, developed recurrent seizures and profound amnesia, and in whom subsequent investigations unexpectedly suggested an autoimmune aetiology.

\section{Case Report}

A 36-year-old right-handed female was referred to our centre for assessment of epileptic seizures and cognitive impairment.

At the age of 33, she had undergone a right (non-dominant) temporal lobectomy for refractory complex partial seizures, performed at another neuroscience centre with an established epilepsy surgery programme. The patient's seizures began at the age of 15 years; there was no history of childhood febrile convulsions. Seizures were characterised by déjà $\mathrm{vu}$, absence, and automatisms, and were thought to arise in the right temporal lobe. MR imaging appearances were equivocal, with right temporal lobe changes thought to represent either sclerosis or a possible dysplastic lesion. Because of the refractory nature of the seizures, pre-operative workup was undertaken, including FDG-PET which showed reduced uptake of tracer in the right temporal lobe. Intracranial EEG (subtemporal strips) confirmed complex partial seizures arising from the lateral right temporal cortex, but there also seemed to be subclinical events arising from the left side. A sodium amytal test performed prior to surgery confirmed that the patient was left-hemisphere dominant for language and that both hemispheres supported memory function.

The post-operative course was initially encouraging, with reduced seizure frequency. However, 3-4 months post-operatively the patient deteriorated with further frequent complex partial seizures. Additionally, she was noted to have symptoms suggestive of both anterograde and retrograde amnesia. By this time she had relocated, and subsequent investigations were undertaken in this neurology department.

Cognitive assessment included administration of cognitive screening instruments which showed impaired performance: on the Mini-Mental State Examination (MMSE) [3], she scored 23/30; on the Addenbrooke's Cognitive Examination-Revised (ACE-R) [4], she scored $74 / 100$, with $12 / 26$ on the memory components; and on the Montreal Cognitive Assessment [5], she scored 23/30 (normal $\geq 26 / 30$ ). On the Repeatable Battery for the Assessment of Neuropsychological Status (RBANS) [6], her delayed memory scores fell within the extremely low range. This impairment was for both verbal and visual material, with a subtle indication of slightly higher levels of delayed recall with visual information (list recall total score $=0$; story recall total score $=1$; figure recall total score $=2$ ). These findings clearly indicated involvement of the unoperated (dominant) hemisphere. Other domains assessed by the RBANS showed the immediate memory to be in the borderline range, attention was low average, whilst language and visuospatial/constructional abilities were relatively preserved (table 1; left-hand column).

Other investigations at this time included MR brain imaging. In addition to the evidence of right temporal lobectomy, this also showed a high signal change in the left temporal lobe involving the hippocampus (fig. 1). CSF analysis showed the presence of oligoclonal bands that were not found in serum. Serological testing revealed a very high titre of antibodies directed against glutamic acid decarboxylase (GAD). In sum, these investigations suggested a diagnosis of anti-GAD limbic encephalitis (LE).

Over the next 2 years, the patient was empirically treated with various immunomodulatory interventions including intravenous methylprednisolone, plasma exchange, and two 
infusions of rituximab, all without obvious clinical improvement in either seizures or cognitive function.

Three years after surgery, prior to embarking on a treatment trial of intravenous immunoglobulin at our centre, the patient was still receiving polytherapy for epileptic seizures (levetiracetam, pregabalin, clonazepam, and lacosamide). On cognitive testing, she now scored $17 / 30$ on the MMSE and $63 / 100$ on the ACE-R with $8 / 26$ on the memory components. Repeating the RBANS, the cognitive profile was little changed, with the delayed memory score still being extremely low (table 1; right-hand column), again affecting both verbal and visual material, with the latter still at slightly higher levels (list recall total score $=$ 2 ; story recall total score $=2$; figure recall total score $=8$ ). Behaviourally, she used external memory aids to record daily events since she had no recall of these after only a brief period of time.

\section{Discussion}

Antibodies to GAD have been associated with various neurological syndromes, including stiff person syndrome, cerebellar ataxia, epilepsy, paraneoplastic syndromes (encephalomyelitis, cerebellar ataxia, and LE), idiopathic LE, and myasthenia gravis [7].

From the epilepsy perspective, in a cohort of 253 epilepsy patients, Liimatainen et al. [8] detected anti-GAD antibodies in 15 patients (5.9 vs. $1.5 \%$ in 200 controls), most of them $(90 \%)$ with temporal lobe epilepsy. In a study of patients with adult-onset (>30 years) temporal lobe epilepsy, anti-GAD antibodies were found in 5 out of 42 , with evidence for pharmacoresistant epilepsy with associated memory impairment and other autoimmune diseases [9]. Temporal lobe epilepsy with anti-GAD antibodies may not therefore be a rare condition, especially amongst the treatment-refractory patients referred for surgical evaluation.

The precise pathogenic sequence of events in our patient remains uncertain; a number of potential explanations exist. It is possible that she had two separate problems, namely mesial temporal sclerosis followed by adult-onset anti-GAD antibody LE. If this is so, it might be speculated whether her temporal lobectomy surgery might have unmasked epitopes which initiated an autoimmune response that produced anti-GAD antibodies and hence LE.

Another possibility is that the entire syndrome was due to anti-GAD LE, albeit very longlived. Malter et al. [10] defined non-paraneoplastic anti-GAD LE as a chronic non-remitting disorder, with antibody titres remaining high after intravenous methylprednisolone. Moreover, none of their patients became seizure free despite intense anti-epileptic drug therapy, unlike the situation in LE associated with voltage-gated potassium channel (VGKC/LGI1) antibodies [10,11]. Furthermore, cognitive impairments did not improve after treatment in anti-GAD LE [12]. In this context, it is of note that the neuropathological examination of our patient's temporal lobectomy specimen found evidence of chronic inflammatory change consisting almost exclusively of $\mathrm{T}$ cells with associated microglial activation, suggesting a chronic encephalitic process in addition to hippocampal sclerosis.

To date there is little information on the efficacy of intravenous immunoglobulin in antiGAD LE [10]. Response has been reported in new-onset focal epilepsy [13], but the chances of success must be doubtful in chronic epilepsy, as in our patient.

This case may illustrate that autoimmune processes, rather than surgery, may cause bilateral hippocampal pathology resulting in profound amnesia, behaviourally akin to that seen in the classic amnesic patient H.M. [14, 15]. Following bilateral anterior temporal lobectomy for intractable seizures with partial hippocampal removal [16], H.M. developed a 
Bonello et al.: Profound Amnesia after Temporal Lobectomy: An Autoimmune Process Resembling Patient H.M.?

profound anterograde amnesia for episodic autobiographical material but with preserved general intelligence, attention, working memory, language and perceptual skills. In contrast, the neuropsychological outcome following unilateral non-dominant hemisphere temporal lobectomy, as undergone by our patient, is usually confined to material-specific (i.e. visual rather than verbal) memory impairments. We recommend that the possibility of anti-GAD LE needs to be considered in all patients with refractory epilepsy of presumed temporal lobe origin, including those being considered for epilepsy surgery, especially those whose clinical course is not typical for mesial temporal sclerosis.

\section{References}

1 Scoville W, Milner B: Loss of recent memory after bilateral hippocampal lesions. J Neurol Neurosurg Psychiatry 1957;20:11-21.

-2 Kapur N, Prevett M: Unexpected amnesia: are there lessons to be learned from cases of amnesia following unilateral temporal lobe surgery? Brain 2003;126:2573-2585.

-3 Folstein MF, Folstein SE, McHugh PR: 'Mini-Mental State'. A practical method for grading the cognitive state of patients for the clinician. J Psychiatr Res 1975;12:189-198.

-4 Mioshi E, Dawson K, Mitchell J, Arnold R, Hodges JR: The Addenbrooke's Cognitive Examination Revised (ACE-R): a brief cognitive test battery for dementia screening. Int J Geriatr Psychiatry 2006;21:1078-1085.

5 Nasreddine ZS, Phillips NA, Bédirian V, et al: The Montreal Cognitive Assessment, MoCA: a brief screening tool for mild cognitive impairment. J Am Geriatr Soc 2005;53:695-699.

-6 Randolph C, Tierney MC, Mohr E, Chase TN: The Repeatable Battery for the Assessment of Neuropsychological Status (RBANS): preliminary clinical validity. J Clin Exp Neuropsychol 1998;20:310319.

7 Saiz A, Blanco Y, Sabater L, et al: Spectrum of neurological syndromes associated with glutamic acid decarboxylase antibodies: diagnostic clues for this association. Brain 2008;131:2553-2563.

-8 Liimatainen S, Peltola M, Sabater L, et al: Clinical significance of glutamic acid decarboxylase antibodies in patients with epilepsy. Epilepsia 2010;51:760-767.

-9 Falip M, Carreno M, Miro J, et al: Prevalence and immunological spectrum of temporal lobe epilepsy with glutamine acid decarboxylase antibodies. Eur J Neurol 2012;19:827-833.

$\$ 10$ Malter MP, Helmstaedter C, Urbach H, Vincent A, Bien CG: Antibodies to glutamic acid decarboxylase define a form of limbic encephalitis. Ann Neurol 2010;67:470-478.

11 Wong SH, Saunders M, Larner AJ, Das K, Hart IK: An effective immunotherapy regimen for VGKC antibodypositive limbic encephalitis. J Neurol Neurosurg Psychiatry 2010;81:1167-1169.

-12 Frisch C, Malter MP, Elger CE, Helmstaedter C: Neuropsychological course of voltage-gated potassium channel and glutamic acid decarboxylase antibody related limbic encephalitis. Eur J Neurol 2013;20:12971304.

13 Marnane M, Vincent A, Hutchinson M: New-onset focal epilepsy with palatal tremor and glutamic acid decarboxylase antibodies responding to intravenous immunoglobulin. J Neurol 2008;255;1603-1604.

14 Moscovitch M: Memory before and after H.M.: an impressionistic historical perspective; in Zeman A, Kapur N, Jones-Gotman M (eds): Epilepsy and Memory. Oxford, Oxford University Press, 2012, pp 19-50.

15 Corkin S: Permanent Present Tense. The Man with no Memory, and What He Taught the World. London, Allen Lane, 2013.

16 Annese J, Schenker-Ahmed NM, Bartsch H, et al: Postmortem examination of patient H.M.'s brain based on histological sectioning and digital 3D reconstruction. Nat Commun 2014;5:3122. 
Neurology

\begin{tabular}{l|l}
\hline DOI: $10.1159 / 000369058$ & $\begin{array}{l}\text { ○ } 2014 \text { S. Karger AG, Basel } \\
\text { www.karger.com/crn }\end{array}$ \\
\hline
\end{tabular}

Bonello et al.: Profound Amnesia after Temporal Lobectomy: An Autoimmune Process Resembling Patient H.M.?

Table 1. Sequential cognitive assessment with the MMSE, ACE-R, and RBANS

\begin{tabular}{lll}
\hline & 6 months after surgery & 3 years after surgery \\
\hline MMSE & $23 / 30$ & $17 / 30$ \\
\hline ACE-R & $74 / 100($ memory $12 / 26)$ & $63 / 100($ memory $8 / 26)$ \\
\hline RBANS & & \\
Immediate memory & $76(5)$ & $69(2)$ \\
Visuospatial/constructional & $96(39)$ & $112(79)$ \\
Language & $101(53)$ & $96(39)$ \\
Attention & $82(12)$ & $97(42)$ \\
Delayed memory & $44(<0.1)$ & $44(<0.1)$ \\
Total scale & $75(8)$ & $79(8)$ \\
\hline
\end{tabular}

For the RBANS, values represent the index scores with percentiles in parentheses.

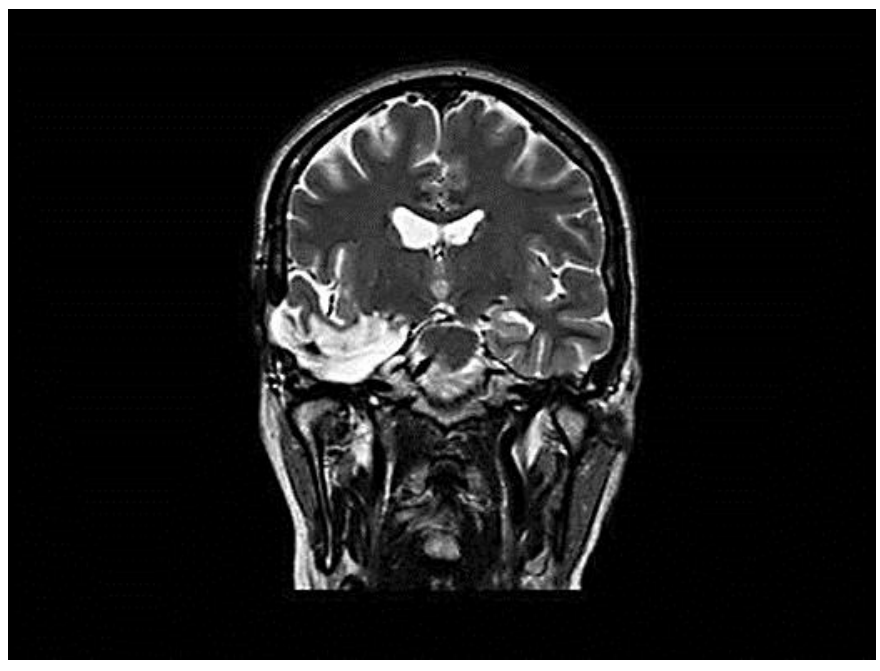

Fig. 1. Coronal $\mathrm{T}_{2}$-weighted $\mathrm{MR}$ imaging showing a surgical cavity in the right middle cranial fossa with gliosis and encephalomalacia in the right temporal lobe. In addition there is a slightly increased signal in the left hippocampus. 